\title{
Modelling the tide effects in groundwater
}

\author{
J. Mls \\ Faculty of Science, Charles University, Czech Republic
}

\begin{abstract}
It is well known that in aquifers in contact with the ocean, sinusoidal fluctuations of groundwater level occur in response to tides. Similar semidiurnal fluctuations have been observed in the piezometric head of confined aquifers without any contact with the ocean. These fluctuations are of interest to hydrogeologists and geophysicists as they indicate that the observed wells are sensitive indicators of the surrounding material deformations either by changes in the surface loading or by applied strain.

The aim of the contribution is to model the pressure-head fluctuations in a leaky confined aquifer as a result of periodic changes of the load originating in the tidal changes of gravity acceleration. The Biot approach was used to derive the governing equations. The aquifer characteristics and the imposed boundary conditions were determined according to the known geology of the particular site where the water level fluctuations were observed. The problem was solved numerically and the obtained results were compared with the measured values.

Keywords: groundwater tides, water level fluctuations, tide potential, tide acceleration, aquifer, solid phase compressibility, Biot's approach.
\end{abstract}

\section{Introduction}

According to Rojstaczer and Agnew [5], high porosity aquifers show high sensitivity to atmospheric loading while high sensitivity to the applied areal strains occurs for low porosity aquifers, and both increase with decreasing compressibility of the solid matrix.

Involving an inertia term, Bodvarsson [2] generalized the Darcy law and gave a theory of strain-induced pressure fluctuations in a well-aquifer system. In his paper, he emphasized the necessity of the wells being connected with a sufficiently confined aquifer. 
Response of the water level in a well to Earth tides and atmospheric loading under unconfined conditions was studied by Rojstaczer and Riley [6]. The authors define physical properties of aquifers to exhibit a significant effect of water level fluctuation: high specific storage, high hydraulic conductivity, and low specific yield.

Boreholes where measurable values of tide oscillation have been found indicate locations where the solid-phase tension of the geological layer does not match the tide forces. At such places, the pressure of the liquid phase takes over a part of the solid-phase tension.

During hydrogeological investigation of the Police basin, situated in northeastern Bohemia, oscillations of water table were found in several boreholes (Krásný et al. [3]). Subsequent more detailed research checked this phenomenon with the effects of atmospheric pressure and groundwater storage due to recharge/discharge processes. The relation was confirmed in all but one of the cases. Water table of the borehole V-34 oscillated in its own way regardless of atmospheric pressure and the pumping process in its neighbourhood. According to the frequency of the water table changes, Krásný et al. [3] came to the conclusion that the observed phenomenon were tidal oscillations of groundwater hydraulic head.

The knowledge of the geology of the investigated region and the knowledge of hydraulic characteristics of the aquifer and surrounding layers make it possible to formulate a simple model of the two phase system and numerically simulate the supposed process. To make this, the Biot [1] approach has been applied connecting the solid-phase elastic deformation with the pressure and flow of the liquid phase within the pore system.

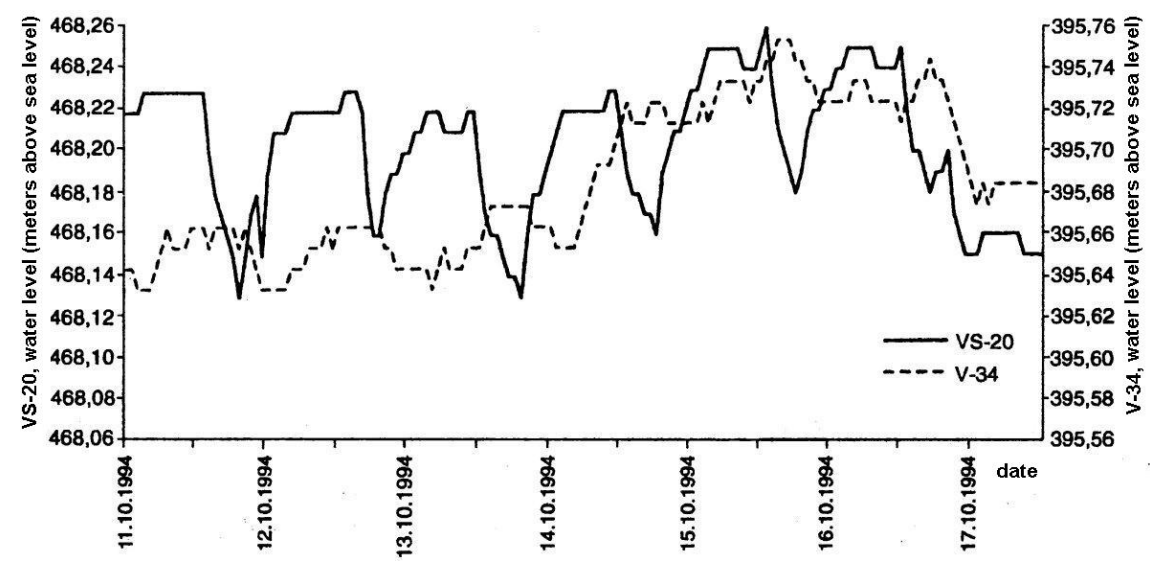

Figure 1: Water level fluctuations indicated in boreholes VS-20 and V-34. 


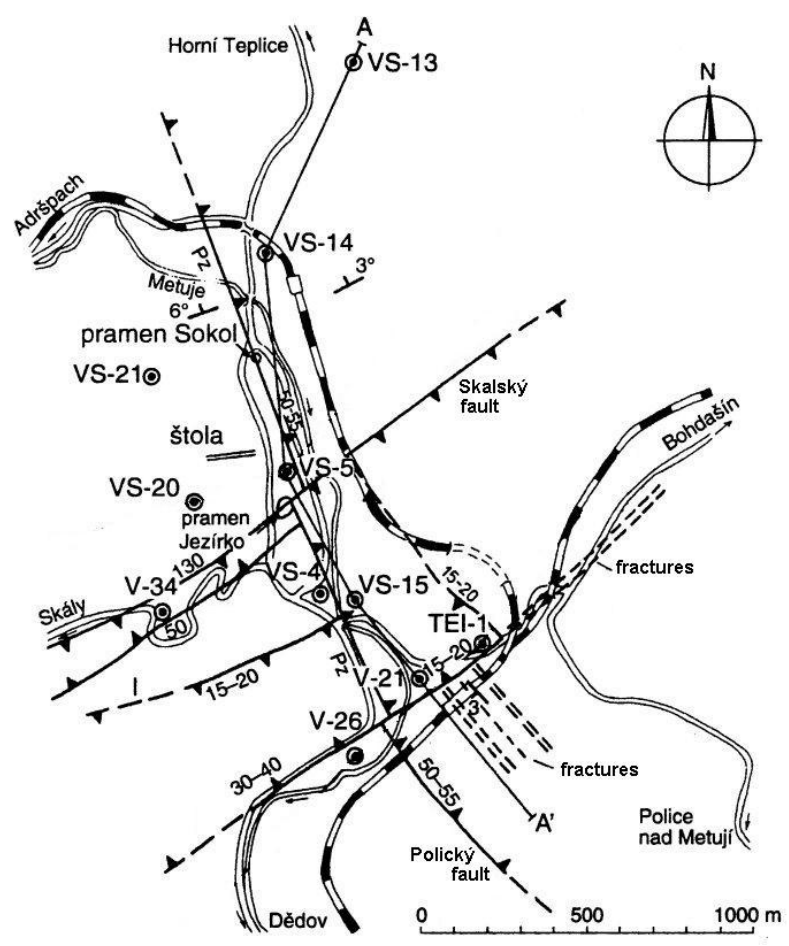

Figure 2: Position of boreholes VS-20 and V-34 and of the faults.

\section{Geological conditions}

Figure 1 shows a water level fluctuation as it was recorded in two boreholes, VS-20 and $\mathrm{V}-34$, in the Police basin. Initially, it was explained by pumping from a water supply borehole lying about 1,5 km north of the measured boreholes. It was later realised that the borehole V-34 was separated from the pumping site by the Skalsk fault. Subsequent test confirmed the assumption that the fault built an impervious obstacle which did not allow for any propagation of hydraulic head changes.

The borehole V-34 is situated in a narrow strip bounded by two faults, Skalsk fault and a shorter parallel one, see Figure 2. The borehole is open to a confined aquifer, the part of which, situated between the planes of the faults, is affected by tension originated in tide forces.

Hydrogeology of the region, the Police basin, and hydraulic characteristics of particular layers are known from the study published by Krsn et al. [3]. This makes it possible to define the conceptual model of the investigated process: the domain, its parameters and the boundary conditions.

The studied domain is the aquifer between the faults. The problem is supposed to be one dimensional defined in horizontal direction normal to the plane of the faults. The coordinate is oriented in the northwest direction and denoted $x$. The domain 


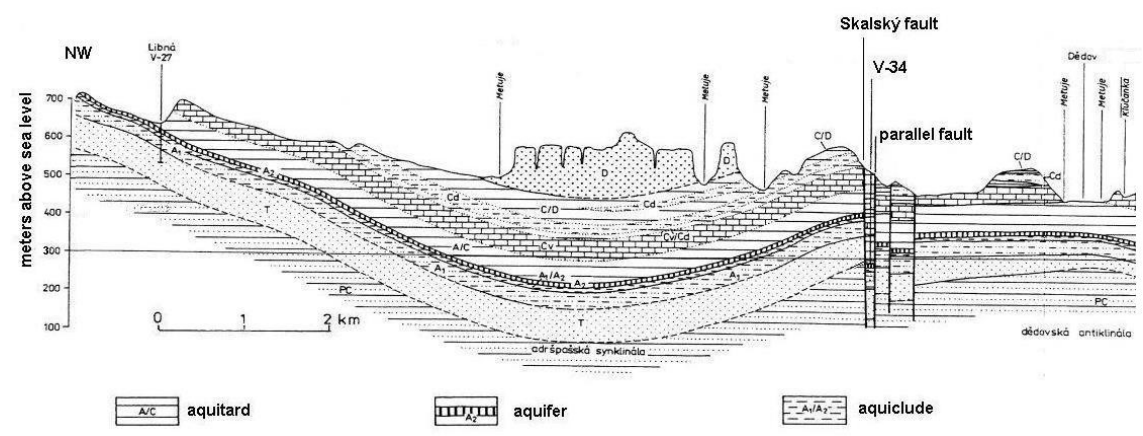

Figure 3: North part of the sectional view AA'.

is then $\Omega=(0, L), L$ being the distance of the faults. In Figure $3, \mathrm{~A}_{2}$ denotes the aquifer, $\mathrm{A}_{1} / \mathrm{A}_{2}$ denotes the underlying layer and $\mathrm{A} / \mathrm{C}$ denotes the overlying layer. The underlying layer is supposed to be an aquiclude and the overlying layer is supposed to be an aquitard. Hydraulic conductivities of these layers, specific storativity of the aquifer and pressure head in the aquifer lying above the layer A/C are known from (Krsn et al. [3]). The region north of the Skalsk fault is elevated about $100 \mathrm{~m}$ above the southern part of the basin. Hence, it is supposed that the part of the massif situated south of the domain $\Omega$, having smaller load, is easier deformable than the northern one. Consequently, it is supposed that the northern part of the basin is rigid and the modelled part of the aquifer is pushed against the plane of the fault.

\section{Governing equations}

Part of the tension arising within geological layers originates in tide acceleration acting upon the mass of the layers. In most cases, the solid phase, due to its toughness, holds most of the tension. There are, however, places where the tide forces affect the liquid phase within the pore space in such a way that the corresponding pressure-head fluctuation is measurable.

Biot [1] published his theory of consolidation which he considered as a process of squeezing water out of a deformable solid-phase body. The Biot theory connects laws of elastic deformation with laws of Darcian flow of fluid in porous media. In this way, it makes it possible to relate the solid-phase tension with liquid-phase pressure and to formulate governing equations of the investigate process.

The initial Biot's assumptions are

$$
\frac{\partial u_{i}}{\partial x_{j}}+\frac{\partial u_{j}}{\partial x_{i}}=\frac{1}{\mu} \tau_{i, j}-\frac{\sigma}{\mu(1+\sigma)} \vartheta \delta_{i, j}+\frac{2 p}{3 H} \delta_{i, j}, \quad i, j=1,2,3,
$$

and

$$
\theta=\theta_{0}+\frac{\vartheta}{3 H}+\frac{p}{R}
$$


Hence, taking into account the equilibrium conditions of a stress field

$$
\frac{\partial \tau_{i, j}}{\partial x_{j}}=0, \quad i=1,2,3,
$$

the resulting equations of the Biot theory are

$$
\mu \Delta u_{i}+\frac{\mu}{1-2 \sigma} \frac{\partial \epsilon}{\partial x_{i}}-\alpha \frac{\partial p}{\partial x_{i}}=0, \quad i=1,2,3,
$$

and

$$
\alpha \frac{\partial \epsilon}{\partial t}+\left(\frac{1}{R}-\frac{\alpha}{H}\right) \frac{\partial p}{\partial t}=\frac{k}{\eta} \Delta p,
$$

where $x_{1}, x_{2}, x_{3}$ are the space coordinates, $t$ is time, $u=\left(u_{1}, u_{2}, u_{3}\right)$ is the solidphase displacement vector, $\tau_{i, j}$ is the tensor of the solid-phase stress, $p$ is the liquid-phase pressure, $\theta$ is the water content, $\delta_{i, j}$ is the Kronecker tensor, $\mu$ is the shear modulus of the solid phase, $\sigma$ is the Poisson ratio, $H$ and $R$ are Biot's coefficients: $1 / H$ is a measure of compressibility of the solid phase for a change in water pressure and $1 / R$ is a measure of the change in water content for a change in water pressure, $k$ is the permeability of the aquifer, $\eta$ is the liquid-phase dynamic viscosity,

$$
\begin{array}{r}
\vartheta=\tau_{j, j}, \\
\epsilon=\frac{\partial u_{j}}{\partial x_{j}},
\end{array}
$$

and

$$
\alpha=\frac{2(1+\sigma) \mu}{3(1-2 \sigma) H}
$$

\section{The solved problem}

According to the assumptions of our model, Equations (4) and (5) are rewritten in the one-dimensional form as

$$
\frac{2 \mu(1-\sigma)}{1-2 \sigma} \Delta u-\alpha \frac{\partial p}{\partial x}=0
$$

and

$$
\alpha \frac{\partial^{2} u}{\partial t \partial x}+\left(\frac{1}{R}-\frac{\alpha}{H}\right) \frac{\partial p}{\partial t}=\frac{k}{\eta} \Delta p .
$$

One-dimensional form of Equation (1) is

$$
\tau=\frac{2 \mu(1-\sigma)}{1-2 \sigma} \frac{\partial u}{\partial x}-\alpha p .
$$


Now, every triplet of functions $\{u(x, t), p(x, t), f(t)\}$ satisfying equation

$$
\frac{2 \mu(1-\sigma)}{1-2 \sigma} \frac{\partial u}{\partial x}-\alpha p=f
$$

satisfies also Equation (9). From Equations (11) and (12), it follows that the function $f$ represents solid-phase stress and that it can be determined provided function $\tau$ is known as a function of time at a single value of coordinate $x$. Consequently, denoting $T(t)$ the tension at a boundary point, it follows from (12)

$$
\frac{\partial^{2} u}{\partial t \partial x}=\frac{\alpha(1-2 \sigma)}{2 \mu(1-\sigma)} \frac{\partial p}{\partial t}+\frac{1-2 \sigma}{2 \mu(1-\sigma)} T^{\prime}(t) .
$$

The last equation makes it possible to exclude the unknown function $u$ from Equation (10) and to solve the resulting second order parabolic equation with the liquid-phase pressure as the unknown function. According to the aquifer and its neighbouring layers, the solved equation is either linear or nonlinear. Solving the problem for a leaky confined aquifer and horizontally oriented domain $\Omega$, it is necessary to introduce a source term and to deal with the continuity equation of the liquid phase in the form

$$
\frac{\partial \theta}{\partial t}+\operatorname{div} v=q,
$$

where $v$ is vector of the liquid-phase flux density and $q$ is the source term, i.e. the volume of water arising in unit volume of the aquifer per unit time. As the hydraulic head of the overlying aquifer as well as the height and the permeability of the aquitard are known, the source term can be determined as a function of the liquid-phase pressure:

$$
q=\frac{k}{\eta}\left(\rho_{w} g+\frac{\hat{p}-p}{Z}\right),
$$

where $\rho_{w}$ is the liquid-phase density, $g$ is the Earth's gravity acceleration, $\hat{p}$ is the liquid-phase pressure at the top of the aquitard and $Z$ is height of the aquitard.

Biot [1] discussed consolidation of saturated soils as a special case of his general equations. He comes to the conclusion that following equation holds for saturated soils:

$$
\alpha=1 \quad \text { and } \quad \frac{H R}{H-\alpha R} \rightarrow \infty .
$$

Hence, the diffusivity coefficient of the liquid-phase pressure is

$$
c=\frac{2 \mu k(1-\sigma)}{\eta(1-2 \sigma)} .
$$

Concerning the boundary conditions, it is supposed that the top of the aquifer is the only part of its boundary that allows for discharge or recharge. Consequently, the boundary $\partial \Omega$ is impervious and the Neumann boundary condition is imposed in the form

$$
\frac{\partial p}{\partial x}(x, t)=0, \quad t>0, x \in \partial \Omega .
$$




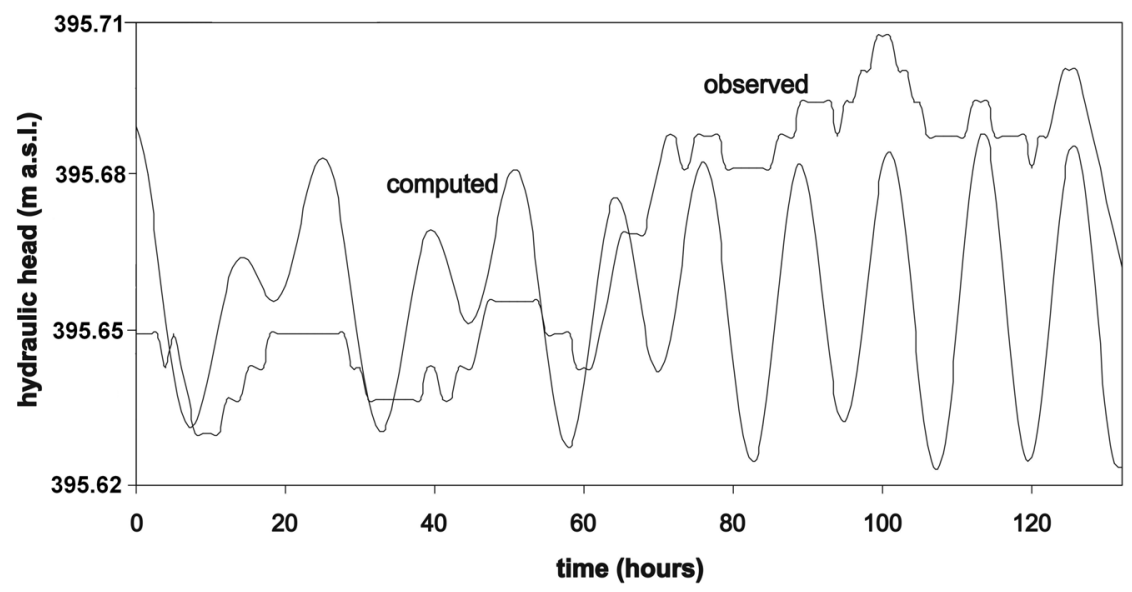

Figure 4: Comparison of observed water level fluctuations with results of a simulation.

\section{Results and discussion}

The tension $T$ is not known in our case. On the other hand, the amplitude of the detected oscillations can be estimated. Hence, function $T$ can be determined by means of the inverse formulation of the problem.

Assuming that the tension $T(t)$ originates in tide acceleration, it can be expressed as

$$
T(t)=M_{N} \gamma(t),
$$

where $M_{N}$ is a constant and $\gamma$ is the projection of the tide acceleration vector into axis $x$ (the normal to the Skalský fault). Choosing a value of $M_{N}$, the forward problem can be solved as described above. Figure 4 presents results of such a simulation.

In this study, the tide acceleration is supposed to be determined by Sun and Moon, the effect of other planets being neglected. Function $\gamma$ was calculated from known coordinates of tide acceleration vector. The code SPZ_SM_01 (Ondovčin [4]), returning values of tide potential and tide-acceleration vector to given time and position on Earth, was used for this purpose.

It can be seen from Figure 4 that in this case, the value of $M_{N}$ was overestimated. On the other hand, the figure shows the most important result of the simulation: there is clear correspondence between the simulated tide effects and the observed data. Consequently, the results confirm the previous conclusion made by Krásný et al. [3].

The oscillations of the computed curve are determined by function $\gamma$ and the value of $M_{N}$ affects just the amplitude. Hence, it is a simple problem to iterate for its proper value. The value $M_{N}=2 \times 10^{8} \mathrm{~kg} / \mathrm{m}^{2}$ was found as the best match of the observed amplitude. 


\section{Acknowledgements}

This paper is based upon work supported by the Grant Agency of the Czech Republic under grant No. 205/07/1311 and by the Ministry of Education of the Czech Republic under grant No. MSM 0021620855. The author is also indebted to the Journal of Geological Sciences for the permission to use Figures 1, 2, and 3.

\section{References}

[1] Biot, M. A., 1941, General theory of three-dimensional consolidation, Journal of Applied Physics, 12, 155-164

[2] Bodvarsson, G., 1970, Confined fluids as strain meters, Journal of Geophysical Research, 75, 14, 2711-2718

[3] Krsn, J., Buchtele, J., ech, S., Hrkal, Z., Jake, P., Kobr, M., Mls, J., Łantrek, J., Łilar, J., Valeka, J., 2002, Hydrogeology of the Police basin: Optimisation of groundwater development and protection, Journal of Geological Sciences, 22, 5-100 (in Czech)

[4] Ondovčin, T., 2007, Description of the code SPZ_SM_01, UK, Prague, (unpublished)

[5] Rojstaczer, S. and Agnew, D. C., 1989, The influence of formation material properties on the response of water levels in Wells to Earth tides and atmospheric loading, Journal of Geophysical Research, 94, B9, 12, 403-12, 411

[6] Rojstaczer, S. and Riley, F. S., 1990, Response of the water level in a well to Earth tides and atmospheric loading under unconfined conditions, Water Resources Research, 26, 1803-1817

[7] van der Kamp, G., and Gale, J. E., 1983, Theory of Erth tide and barometric effects in porous formations with compressible grains, Water Resources Research, 19, 538-544 